NISSUNA UMANA INVESTIGAZIONE SI PUO DIMANDARE VERA SCIENZIA S'ESSA NON PASSA PER LE MATEMATICHE DIMOSTRAZIONI LEONARDO DA VINCI

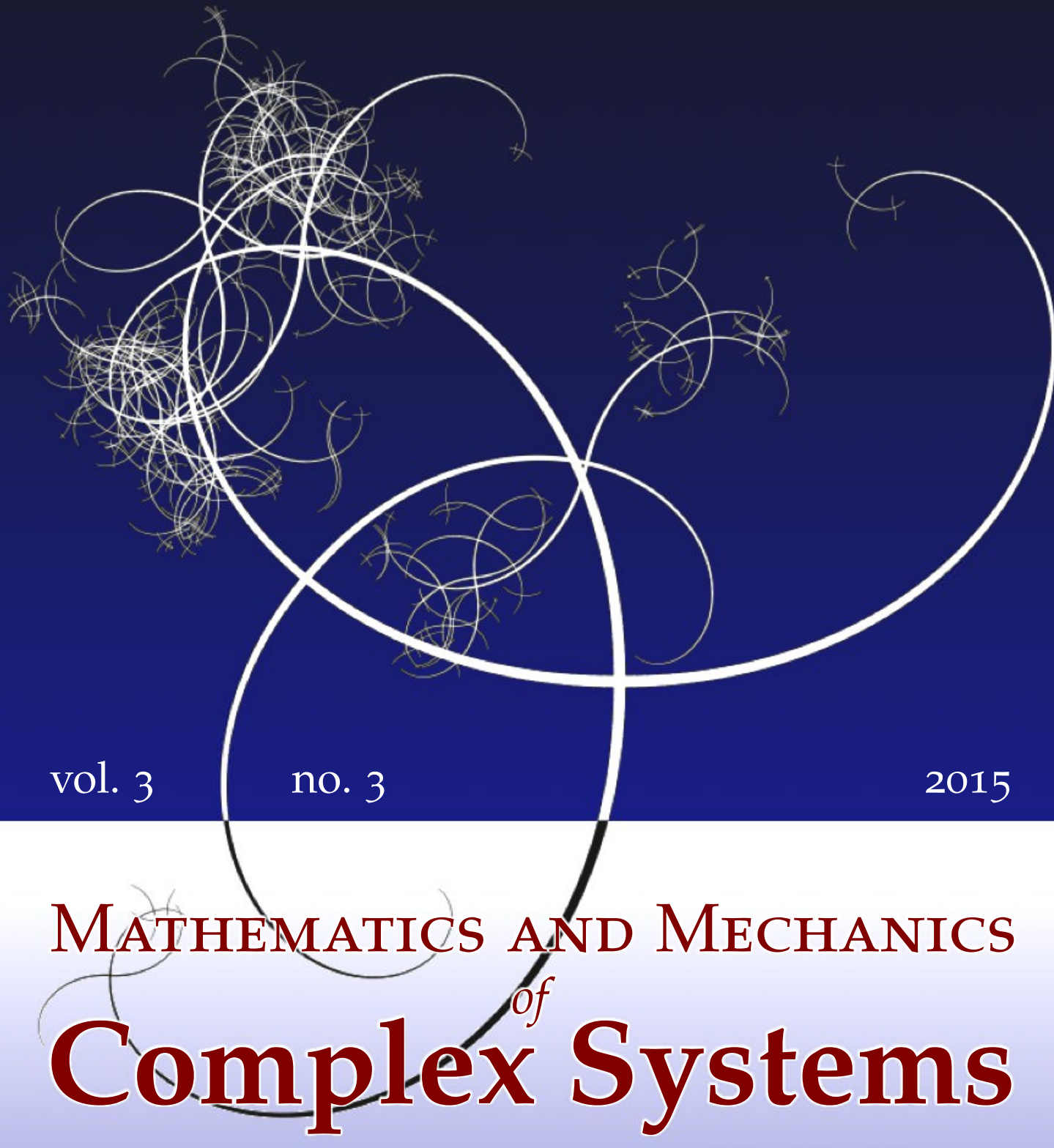

Holm Altenbach and Victor A. EREmeyeV

ON THE CONSTITUTIVE EQUATIONS

OF VISCOELASTIC MICROPOLAR PLATES AND SHELLS

OF DIFFERENTIAL TYPE 


\title{
ON THE CONSTITUTIVE EQUATIONS \\ OF VISCOELASTIC MICROPOLAR PLATES AND SHELLS OF DIFFERENTIAL TYPE
}

\author{
Holm Altenbach and Victor A. EREmeyeV
}

\begin{abstract}
Within the framework of the micropolar theory of continuum we discuss the constitutive equations of viscoelastic micropolar thin-walled structures, i.e. viscoelastic micropolar plates and shells. Starting from the linear viscoelastic micropolar continuum and using the correspondence principle of the linear viscoelasticity we extend the procedure of reduction of three-dimensional equilibrium equations of elastic shell-like solids to the case of viscoelastic behavior. We restricted ourselves by constitutive equations of differential type. In other words, we consider both 2D and 3D constitutive equations which are linear dependencies between certain set of time derivatives of stress and strain measures.
\end{abstract}

\section{Introduction}

The model of the Cosserat or micropolar continuum has recently found new applications in the modeling of the behavior of materials and structures with complex inner structure; see [Eremeyev et al. 2013] and references therein. Since the seminal paper [Ericksen and Truesdell 1958] the Cosserat model has found numerous applications in construction of various generalized models for beams, plates, and shells; see the review and bibliography in [Altenbach et al. 2009]. Within the framework of the direct approach of Ericksen and Truesdell, the shell is modeled as a deformable surface at each point of which a set of directors additionally is attached. In the literature are also known theories of plates and shells based on the reduction of three-dimensional micropolar continuum equations; see for example [Eringen 1999; Reissner 1977; Sargsyan 2011; Zubov 2009; Steinberg and Kvasov 2013; Altenbach et al. 2009], where various averaging procedures in the thickness direction together with the approximation of the displacements and rotations or the force and moment stresses in the thickness direction are applied. In major cases these considerations are restricted by the elastic behavior. For the linear theory of

\section{Communicated by Francesco dell'Isola.}

MSC2010: primary 74A35; secondary 74K20, 74K25, 74D05, 74A20.

Keywords: micropolar plate, Cosserat continuum, viscoelasticity, through-the-thickness integration, constitutive equations. 
viscoelasticity the application of the correspondence principle gives the possibility to derive the theory of viscoelasticity in the case of thin-walled structures such as plates and shells.

In this paper we extend the through-the thickness integration procedure applied in [Altenbach and Eremeyev 2009] to viscoelastic micropolar plates and shells. The interest to the theory of viscoelastic micropolar thin-walled structures is based on prospective applications of this theory to the mechanics of plates and shells made of materials with complex inner structure, for example cellular materials and foams for which the micropolar model is used; see [Diebels and Steeb 2003; Lakes 1986] among others.

We consider here the variant of plates and shell theory based on six kinematically independent variables, namely translations and rotations. This theory can be derived using a direct approach [Eremeyev et al. 2013; Eremeyev and Zubov 2008; Rubin 2000] or based on the reduction of the three-dimensional motion equations [Chróścielewski et al. 2004; Libai and Simmonds 1998]. The mathematical study of boundary-value problems was performed in [Bîrsan and Neff 2013; 2014; Eremeyev and Lebedev 2011], while various solutions and finite-element calculations are presented in [Chróścielewski et al. 2004; 2010; 2011; Eremeyev and Zubov 2008].

\section{Basic relations of the viscoelastic Cosserat continuum.}

Following [Eringen 1999] we recall the governing equations of the linear micropolar viscoelasticity. Let the micropolar body occupies the domain $\mathcal{V} \in \mathbb{R}^{3}$. The infinitesimal deformations of the micropolar media are described by two vectorial fields. The first one is the vector of translation $\boldsymbol{u}$ and the second field is the vector of microrotation $\boldsymbol{\vartheta}$ given as vector-functions of the position vector $\boldsymbol{x}$ and time $t$. From the physical point of view, $\boldsymbol{u}$ describes an displacement of the particle of a micropolar body while $\vartheta$ corresponds to the particle rotation. The quasistatic deformations of a micropolar body are described by the equilibrium equations

$$
\nabla \cdot \boldsymbol{\sigma}+\rho \boldsymbol{f}=\mathbf{0}, \quad \nabla \cdot \boldsymbol{\mu}+\boldsymbol{\sigma}_{\times}+\rho \boldsymbol{\ell}=\mathbf{0}, \quad \boldsymbol{x} \in \mathcal{V},
$$

where $\nabla$ is the three-dimensional nabla operator, $f$ and $\ell$ are the mass forces and the mass couples vectors, $\rho$ is the density, $\sigma$ and $\mu$ are the stress tensor and the couple stress tensor, respectively, and $\sigma_{\times}$denotes the vectorial invariant of the second-order tensor $\boldsymbol{\sigma}$, see [Lebedev et al. 2010; Wilson 1901]. Equation (1) represents the local form of the balance of momentum while Eq. $(1)_{2}$ is the balance of moment of momentum.

The static boundary conditions have the following form

$$
\boldsymbol{n} \cdot \boldsymbol{\sigma}=\boldsymbol{t}^{0}, \quad \boldsymbol{n} \cdot \boldsymbol{\mu}=\boldsymbol{m}^{0} \quad \text { at } S_{f} .
$$


Here $\boldsymbol{t}^{0}$ and $\boldsymbol{m}^{0}$ are the surface forces and the surface couples acting on the part of the surface $S_{f}$ of the micropolar body, $S=S_{u} \cup S_{f} \equiv \partial \mathcal{V}$. The kinematic boundary conditions consist of the following relations

$$
\boldsymbol{u}=\boldsymbol{u}^{0}, \quad \vartheta=\vartheta^{0} \quad \text { at } S_{u},
$$

where $\boldsymbol{u}^{0}$ and $\vartheta^{0}$ are given functions at $S_{u}$. Let us note that since the displacements and rotations are independent kinematic variables, Dirichlet and Neumann boundaries for the displacements and rotations are different, in general. As a result, the kinematic and static boundary conditions take the form

$$
\begin{array}{llll}
\boldsymbol{n} \cdot \boldsymbol{\sigma}=\boldsymbol{t}^{0} & \text { at } S_{f}, & \boldsymbol{u}=\boldsymbol{u}^{0} & \text { at } S_{u}, \\
\boldsymbol{n} \cdot \boldsymbol{\mu}=\boldsymbol{m}^{0} & \text { at } S_{m}, & \boldsymbol{\vartheta}=\boldsymbol{\vartheta}^{0} & \text { at } S_{\theta},
\end{array}
$$

where $S=S_{u} \cup S_{f}=S_{m} \cup S_{\theta}$ are two decompositions of $S$. For simplicity in what follows we use the same boundaries that is $S_{u}=S_{\theta}$ and $S_{f}=S_{m}$. Obviously, other mixed boundary conditions are also possible [Eremeyev et al. 2013].

The linear strain measures, i.e. the linear stretch tensor $\boldsymbol{\varepsilon}$ and the linear wryness tensor $\mathfrak{x}$, are given by the relations

$$
\boldsymbol{\varepsilon}=\nabla \boldsymbol{u}+\boldsymbol{\vartheta} \times \mathbf{I}, \quad \boldsymbol{x}=\nabla \vartheta
$$

where $\mathbf{I}$ is the unit three-dimensional tensor and $\times$ the cross product.

Let us consider the viscoelastic micropolar isotropic material. For the sake of simplicity we restrict ourselves by the constitutive equations of the differential type. This means that the constitutive equations are

$$
\begin{aligned}
P_{0}\left(\partial_{t}\right) \boldsymbol{\sigma} & =P_{1}\left(\partial_{t}\right) \boldsymbol{\varepsilon}+P_{2}\left(\partial_{t}\right) \boldsymbol{\varepsilon}^{T}+P_{3}\left(\partial_{t}\right) \mathbf{I} \operatorname{tr} \boldsymbol{\varepsilon}, \\
Q_{0}\left(\partial_{t}\right) \boldsymbol{\mu} & =Q_{1}\left(\partial_{t}\right) \boldsymbol{x}+Q_{2}\left(\partial_{t}\right) \boldsymbol{x}^{T}+Q_{3}\left(\partial_{t}\right) \mathbf{I} \operatorname{tr} \boldsymbol{x} .
\end{aligned}
$$

In (6) and (7) $\partial_{t}$ stands for the derivative with respect to time; $P_{0}, P_{1}, P_{2}, P_{3}, Q_{0}$, $Q_{1}, Q_{2}, Q_{3}$ are the polynomials.

Using the Laplace transform of a function $f(t)$, given by

$$
\bar{f}(p)=\int_{0}^{\infty} f(t) e^{-p t} \mathrm{~d} t,
$$

the constitutive equations (6) and (7) become

$$
\overline{\boldsymbol{\sigma}}=\lambda \mathbf{I} \operatorname{tr} \overline{\boldsymbol{\varepsilon}}+\mu \overline{\boldsymbol{\varepsilon}}^{T}+(\mu+\kappa) \overline{\boldsymbol{\varepsilon}}, \quad \overline{\boldsymbol{\mu}}=\alpha \mathbf{I} \operatorname{tr} \overline{\mathbf{\boldsymbol { x }}}+\beta \overline{\mathbf{x}}^{T}+\gamma \overline{\mathbf{x}},
$$

where

$$
\lambda=\frac{P_{3}(p)}{P_{0}(p)}, \quad \mu=\frac{P_{2}(p)}{P_{0}(p)}, \quad \kappa=\frac{P_{1}(p)-P_{2}(p)}{P_{0}(p)},
$$




$$
\alpha=\frac{Q_{3}(p)}{Q_{0}(p)}, \quad \beta=\frac{Q_{2}(p)}{Q_{0}(p)}, \quad \gamma=\frac{Q_{1}(p)}{Q_{0}(p)}
$$

are the Laplace transforms of the relaxation functions of the viscoelastic micropolar material. In fact, (8) with (9), (10) coincide up to notations with the constitutive equations of an isotropic linear micropolar solid, see [Eringen 1999].

Substituting (8) into (1) one may derive the equilibrium equations in terms of the kinematical fields $\overline{\boldsymbol{u}}$ and $\bar{\vartheta}$. For homogeneous micropolar bodies these equations are

$$
\begin{gathered}
(\lambda+\mu) \nabla \nabla \cdot \overline{\boldsymbol{u}}+(\mu+\kappa) \nabla \cdot \nabla \overline{\boldsymbol{u}}+\kappa \nabla \times \overline{\boldsymbol{\vartheta}}+\rho \overline{\boldsymbol{f}}=\mathbf{0}, \\
(\alpha+\beta) \nabla \nabla \cdot \overline{\boldsymbol{\vartheta}}+\gamma \nabla \cdot \nabla \overline{\boldsymbol{\vartheta}}+\kappa \nabla \times \overline{\boldsymbol{u}}-2 \kappa \overline{\boldsymbol{\vartheta}}+\rho \overline{\boldsymbol{\ell}}=\mathbf{0} .
\end{gathered}
$$

Remark. In a similar manner other linear viscoelastic constitutive equations (integral equation, complex moduli equations) can be introduced and applied to the representation of the viscoelastic micropolar behavior.

In what follows we consider the reduction of three-dimensional equilibrium equations to the two-dimensional ones and discuss the corresponding two-dimensional constitutive equations.

\section{Micropolar plate and shell equations}

Within the framework of the linear theory of micropolar plates and shells, also called the six-parameter theory of shells, we consider a micropolar shell as a two-dimensional Cosserat continuum, i.e., as a deformable surface $\mathcal{M}$ with six degrees of freedom. Each material point of the surface is kinematically similar to an infinitesimal rigid body with three translational and three rotational degrees of freedom. Hence, the deformations of the micropolar plate or shell are described by the translation vector $\boldsymbol{v}$ and the rotation vector $\boldsymbol{\theta}$ which are defined at the base surface $\mathcal{M}$. Using the direct approach the basics of the linear theory of micropolar shells are summarized in [Eremeyev and Zubov 2008; Lebedev et al. 2010]; see also Appendix D in [Eremeyev et al. 2013]. The governing equations of the micropolar shells and plates coincide with the relations of the general 6-parameter nonlinear shell theory presented in [Chróścielewski et al. 2004; Libai and Simmonds 1998] in the case of small deformations derived using the through-the-thickness integration procedure.

The balance of momentum and the balance of moment of momentum are formulated as follows

$$
\nabla_{s} \cdot \mathbf{T}+\boldsymbol{q}=\mathbf{0}, \quad \nabla_{s} \cdot \mathbf{M}+\mathbf{T}_{\times}+\boldsymbol{c}=\mathbf{0},
$$

where $\boldsymbol{q}$ and $\boldsymbol{c}$ are the surface loads (forces and moments), $\mathbf{T}$ and $\mathbf{M}$ are the resultant force stress and couple stress tensors, and $\nabla_{s}$ is the surface nabla differential 
operator. The tensors $\mathbf{T}$ and $\mathbf{M}$ have the properties

$$
\boldsymbol{n} \cdot \mathbf{T}=\mathbf{0}, \quad \boldsymbol{n} \cdot \mathbf{M}=\mathbf{0},
$$

where $\mathbf{n}$ is the unit vector of normal to $\mathcal{M}$. Hence, $\mathbf{T}$ and $\mathbf{M}$ take the form

$$
\mathbf{T}=T_{\alpha \beta} \boldsymbol{i}^{\alpha} \otimes \boldsymbol{i}^{\beta}+T_{\alpha 3} \boldsymbol{i}^{\alpha} \otimes \boldsymbol{n}, \quad \mathbf{M}=M_{\alpha \beta} \boldsymbol{i}^{\alpha} \otimes \boldsymbol{i}^{\beta}+M_{\alpha 3} \boldsymbol{i}^{\alpha} \otimes \boldsymbol{n} \quad(\alpha, \beta=1,2),
$$

where $\boldsymbol{i}^{\alpha}$ are the base vectors on the shell surface. Obviously, in this theory of shells the action of the drilling moment $M_{\alpha 3}$ is taken into account. For example, such possibility may be useful to describe the interaction of the shell and the rigid body or for the description of the deformations of multifolded plates, see [Chróścielewski et al. 2004].

The static and kinematic boundary conditions take the form

$$
\begin{aligned}
\boldsymbol{v} \cdot \mathbf{T} & =\boldsymbol{t}_{s}^{*}, & \boldsymbol{v} \cdot \mathbf{M} & =\boldsymbol{m}_{s}^{*} & & \text { along } \mathcal{C}_{f}, \\
\boldsymbol{v} & =\boldsymbol{v}^{0}, & \boldsymbol{\theta} & =\boldsymbol{\theta}^{0} & & \text { along } \mathcal{C}_{u} .
\end{aligned}
$$

Here $\boldsymbol{v}$ is the vector of the unit normal to $\mathcal{C}_{f}, \boldsymbol{v} \cdot \boldsymbol{n}=0, \boldsymbol{t}_{s}^{*}$ and $\boldsymbol{m}_{s}^{*}$ are external force and couple vectors acting along the boundary $\mathcal{C}_{f}$, while $\boldsymbol{v}^{0}$ and $\boldsymbol{\theta}^{0}$ are given functions describing the displacements and rotations of the boundary $\mathcal{C}_{u}$, respectively, $\mathcal{C}=\mathcal{C}_{u} \cup \mathcal{C}_{f} \equiv \partial \mathcal{M}$. For simplicity we use here again the same boundaries for Dirichlet and Neumann conditions for displacements and rotations.

The linear surface strain measures are

$$
\boldsymbol{\epsilon}=\nabla_{s} \boldsymbol{v}+\mathbf{A} \times \boldsymbol{\theta}, \quad \boldsymbol{\kappa}=\nabla_{s} \boldsymbol{\theta},
$$

where $\mathbf{A} \equiv \mathbf{I}-\boldsymbol{n} \otimes \boldsymbol{n}$ is the two-dimensional or surface unit tensor.

We restrict ourselves again by the constitutive equations for $\mathbf{T}$ and $\mathbf{M}$ of differential type. We assume the following relations

$$
\begin{aligned}
& A_{0}\left(\partial_{t}\right) \mathbf{T}=\mathbf{A} A_{1}\left(\partial_{t}\right) \operatorname{tr} \boldsymbol{\epsilon}_{\|}+A_{2}\left(\partial_{t}\right) \boldsymbol{\epsilon}_{\|}^{T}+A_{3}\left(\partial_{t}\right) \boldsymbol{\epsilon}_{\|}+A_{4}\left(\partial_{t}\right) \boldsymbol{\epsilon} \cdot \boldsymbol{n} \otimes \boldsymbol{n}, \\
& B_{0}\left(\partial_{t}\right) \mathbf{M}=\mathbf{A} B_{1}\left(\partial_{t}\right) \operatorname{tr} \boldsymbol{\kappa}_{\|}+B_{2}\left(\partial_{t}\right) \boldsymbol{\kappa}_{\|}^{T}+B_{3}\left(\partial_{t}\right) \boldsymbol{\kappa}_{\|}+B_{4}\left(\partial_{t}\right) \boldsymbol{\kappa} \cdot \boldsymbol{n} \otimes \boldsymbol{n},
\end{aligned}
$$

Here $\boldsymbol{\epsilon}_{\|}=\boldsymbol{\epsilon} \cdot \mathbf{A}, \boldsymbol{\kappa}_{\|}=\boldsymbol{\kappa} \cdot \mathbf{A}$, and $A_{k}(p), B_{k}(p), k=0,1,2,3$, are polynomials.

We call the constitutive equations for the shell (17) and (18) the Maxwell-type, if $A_{0}=1+a_{0} p, B_{0}=1+b_{0} p$, where $1 / a_{0}$ and $1 / b_{0}$ are the relaxation time for the stress resultants and for couple stresses, respectively, and if other polynomials are constants. We call (17) and (18) the Voigt-type constitutive equation, if $A_{0}=B_{0}=1$ while other polynomials are linear functions of $p$.

Using the Laplace transform these constitutive equations become

$$
\begin{gathered}
\overline{\mathbf{T}}=\alpha_{1} \mathbf{A} \operatorname{tr} \overline{\boldsymbol{\epsilon}}_{\|}+\alpha_{2} \overline{\boldsymbol{\epsilon}}_{\|}^{T}+\alpha_{3} \overline{\boldsymbol{\epsilon}}_{\|}+\alpha_{4} \overline{\boldsymbol{\epsilon}} \cdot \boldsymbol{n} \otimes \boldsymbol{n}, \\
\overline{\mathbf{M}}=\beta_{1} \mathbf{A} \operatorname{tr} \overline{\boldsymbol{\kappa}}_{\|}+\beta_{2} \overline{\boldsymbol{\kappa}}_{\|}^{T}+\beta_{3} \overline{\boldsymbol{\kappa}}_{\|}+\beta_{4} \overline{\boldsymbol{\kappa}} \cdot \boldsymbol{n} \otimes \boldsymbol{n},
\end{gathered}
$$


where

$$
\alpha_{i}=\frac{A_{i}(p)}{A_{0}(p)}, \quad \beta_{i}=\frac{B_{i}(p)}{B_{0}(p)}, \quad i=1,2,3,4 .
$$

The final step in the theory of micropolar viscoelastic shells is the construction of the polynomials $A_{k}$ and $B_{k}$ on the base of the dimension reduction of the threedimensional continuum or by experiments. Further we consider the derivation of the relaxation functions $\alpha_{i}$ and $\beta_{i}$ using the through-the-thickness integration procedure presented in [Altenbach and Eremeyev 2009; 2010].

\section{Reduction of constitutive equations from three to two dimensions}

For simplicity let us consider the undeformed plane geometry; i.e., we restrict ourselves to the theory of plates. The case of shells can be discussed by similar way. Using the correspondence principle [Christensen 1971] from [Altenbach and Eremeyev 2009] it follows

$$
\overline{\mathbf{T}}=\langle\mathbf{A} \cdot \overline{\boldsymbol{\sigma}}\rangle, \quad \overline{\mathbf{M}}=\langle\mathbf{A} \cdot \overline{\boldsymbol{\mu}}\rangle-\langle\mathbf{A} \cdot z \overline{\boldsymbol{\sigma}} \times \boldsymbol{n}\rangle,
$$

where $z$ is the coordinate along the thickness coordinate axis. The averaging operator $\langle\cdot\rangle$ is defined as follows

$$
\langle f\rangle=\int_{-h / 2}^{h / 2} f(z) \mathrm{d} z .
$$

For derivation of (21) we refer to the Appendix.

As a result the two-dimensional material parameters are given by the relations

$$
\begin{gathered}
\bar{\alpha}_{1}=\Lambda h \equiv \frac{\bar{\lambda}(2 \bar{\mu}+\bar{\kappa})}{\bar{\lambda}+2 \bar{\mu}+\bar{\kappa}} h, \quad \bar{\alpha}_{2}=\bar{\mu} h, \quad \bar{\alpha}_{3}=(\bar{\mu}+\bar{\kappa}) h, \quad \bar{\alpha}_{4}=(\bar{\mu}+\bar{\kappa}) h, \\
\bar{\beta}_{1}=\bar{\alpha} h-\bar{\mu} \frac{h^{3}}{12}, \quad \bar{\beta}_{2}=\bar{\beta} h-\Lambda \frac{h^{3}}{12}, \quad \bar{\beta}_{3}=\bar{\gamma} h+(2 \bar{\mu}+\bar{\kappa}+\Lambda) \frac{h^{3}}{12}, \quad \beta_{4}=\bar{\gamma} h,
\end{gathered}
$$

where $h$ is the plate thickness.

From (22) and (9) it follows the relations for the tangential relaxation functions

$$
\frac{P_{3}}{P_{0}} \frac{P_{1}+P_{2}}{P_{1}+P_{2}+P_{3}} h=\frac{A_{1}}{A_{0}}, \quad \frac{P_{2}}{P_{0}} h=\frac{A_{2}}{A_{0}}, \quad \frac{P_{1}}{P_{0}} h=\frac{A_{3}}{A_{0}}=\frac{A_{4}}{A_{0}} .
$$

It is clear that determination of the polynomials $A_{k}(p)$ from the latter equations can not be performed uniquely. Solving (24) results in

$$
\begin{array}{ll}
A_{0}=P_{0}\left(P_{1}+P_{2}+P_{3}\right), & A_{1}=P_{3}\left(P_{1}+P_{2}\right) h, \\
A_{2}=P_{2}\left(P_{1}+P_{2}+P_{3}\right) h, & A_{3}=A_{4}=P_{1}\left(P_{1}+P_{2}+P_{3}\right) h .
\end{array}
$$


In a similar way from (23) and (10) we obtain the equations for the bending relaxation functions

$$
\begin{aligned}
& \frac{B_{1}}{B_{0}}=\frac{Q_{3}}{Q_{0}} h-\frac{P_{2}}{P_{0}} \frac{h^{3}}{12}, \quad \frac{B_{2}}{B_{0}}=\frac{Q_{2}}{Q_{0}} h-\frac{P_{3}\left(P_{1}+P_{2}\right)}{P_{0}\left(P_{1}+P_{2}+P_{3}\right)} \frac{h^{3}}{12}, \\
& \frac{B_{3}}{B_{0}}=\frac{Q_{1}}{Q_{0}} h+\frac{\left(P_{1}+P_{2}\right)\left(P_{1}+P_{2}+2 P_{3}\right)}{P_{0}\left(P_{1}+P_{2}+P_{3}\right)} \frac{h^{3}}{12}, \quad \frac{B_{4}}{B_{0}}=\frac{Q_{1}}{Q_{0}} h .
\end{aligned}
$$

From this it follows that

$$
\begin{aligned}
& B_{0}=P_{0} Q_{0}\left(P_{1}+P_{2}+P_{3}\right), \\
& B_{1}=\left(Q_{3} P_{0} h-P_{2} Q_{0} \frac{h^{3}}{12}\right)\left(P_{1}+P_{2}+P_{3}\right), \\
& B_{2}=Q_{2} P_{0}\left(P_{1}+P_{2}+P_{3}\right) h-P_{3} Q_{0}\left(P_{1}+P_{2}\right) \frac{h^{3}}{12}, \\
& B_{3}=Q_{1} P_{0}\left(P_{1}+P_{2}+P_{3}\right) h+Q_{0}\left(P_{1}+P_{2}\right)\left(P_{1}+P_{2}+2 P_{3}\right) \frac{h^{3}}{12}, \\
& B_{4}=Q_{1} P_{0}\left(P_{1}+P_{2}+P_{3}\right) h .
\end{aligned}
$$

Hence, the differential operators in the constitutive equation for the resultant stress and couple stress tensors $\mathbf{T}$ and $\mathbf{M}$ are more complicated than in the threedimensional case because the order of derivatives of $A_{i}$ and $B_{i}$ is higher than the order of $P_{k}$ and $Q_{k}$, in general. This means that the viscoelastic properties of the two-dimensional structure are more complicated then their three-dimensional counterparts.

Remark. In the case of shells the stress resultant and couple stress tensors depend on $\sigma$ as follows:

$$
\overline{\mathbf{T}}=\left\langle(\mathbf{A}-z \mathbf{B})^{-1} \cdot \overline{\boldsymbol{\sigma}}\right\rangle, \quad \overline{\mathbf{M}}=\left\langle(\mathbf{A}-z \mathbf{B})^{-1} \cdot \overline{\boldsymbol{\mu}}\right\rangle-\left\langle(\mathbf{A}-z \mathbf{B})^{-1} \cdot z \overline{\boldsymbol{\sigma}} \times \boldsymbol{n}\right\rangle,
$$

where $\mathbf{B}=-\nabla_{S} \boldsymbol{n}$ is the curvature tensor of the base shell surface, and $\langle\cdot\rangle$ takes a more complex form; see [Lebedev et al. 2010] for details. This means that for a curved surface one should obtain more complicated relaxation properties for twodimensional theories of shell and plate.

\section{Examples}

Let us consider the simple case of the viscoelastic micropolar constitutive equations and the corresponding two-dimensional equations.

5.1. Maxwell model. For a Maxwell-type model the polynomials in (6) and (7) have the form

$$
\begin{array}{rlrl}
P_{0} & =1+p_{0} p, & P_{1}, P_{2}, P_{3} & =\text { const }, \\
Q_{0} & =1+q_{0} p, & Q_{1}, Q_{2}, Q_{3}=\text { const } .
\end{array}
$$


For the stress resultant tensor $\mathbf{T}$ we obtain the constitutive equation of Maxwelltype, i.e. $A_{0}$ is the linear polynomial only, while the $A_{1}, A_{2}, A_{3}$, and $A_{4}$ are constants. For the couple stress tensor $\mathbf{M}$ we obtain more complicated form of constitutive equations. The simplification is possible if $p_{0}=q_{0}=1 / \tau$. In this case we use the polynomials

$$
\begin{aligned}
& B_{0}=P_{0}\left(P_{1}+P_{2}+P_{3}\right), \\
& B_{1}=\left(Q_{3} h-P_{2} \frac{h^{3}}{12}\right)\left(P_{1}+P_{2}+P_{3}\right), \\
& B_{2}=Q_{2}\left(P_{1}+P_{2}+P_{3}\right) h-P_{3}\left(P_{1}+P_{2}\right) \frac{h^{3}}{12}, \\
& B_{3}=Q_{1}\left(P_{1}+P_{2}+P_{3}\right) h+\left(P_{1}+P_{2}\right)\left(P_{1}+P_{2}+2 P_{3}\right) \frac{h^{3}}{12}, \\
& B_{4}=Q_{1}\left(P_{1}+P_{2}+P_{3}\right) h,
\end{aligned}
$$

where $B_{0}$ is a polynomial while $B_{1}, B_{2}, B_{3}$, and $B_{4}$ are constants. Thus, we obtain the Maxwell-type two-dimensional constitutive equations in the case when the relaxation times for the stress and couple stress coincide, i.e., when $P_{0} \equiv Q_{0}$, and we have one relaxation time $\tau$. In the general case we obtain more general constitutive equation for $\mathbf{M}$.

5.2. Voigt model. Assume the polynomials in (6) and (7) have the form

$$
\begin{array}{llll}
P_{0}=1, & P_{1}=P_{1}^{0}\left(1+p_{1} p\right), & P_{2}=P_{2}^{0}\left(1+p_{2} p\right), & P_{3}=P_{3}^{0}\left(1+p_{3} p\right), \\
Q_{0}=1, & Q_{1}=Q_{1}^{0}\left(1+q_{1} p\right), & Q_{2}=Q_{2}^{0}\left(1+q_{2} p\right), & Q_{3}=Q_{3}^{0}\left(1+q_{3} p\right) .
\end{array}
$$

Here the corresponding polynomials $A_{i}, i=1,2,3,4$, are linear functions of $p$. This means that for $\mathbf{T}$ we have the viscoelastic equations which are similar to the standard viscoelastic model [Christensen 1971]. For M we again obtain a more complicated model because in the general case. $B_{0}$ is a linear function while $B_{1}$, $B_{2}, B_{3}$, and $B_{4}$ are quadratic functions of $p$.

A simplification of the two-dimensional constitutive equation for $\mathbf{M}$ is possible if we assume $p_{1}=p_{2}=p_{3}=p_{4}=q_{1}=q_{2}=q_{3}=q_{4}=1 / \tau$. In this case we have

$$
\begin{aligned}
& B_{0}=\left(P_{1}^{0}+P_{2}^{0}+P_{3}^{0}\right), \\
& B_{1}=\left(Q_{3}^{0} h-P_{2}^{0} \frac{h^{3}}{12}\right)\left(P_{1}^{0}+P_{2}^{0}+P_{3}^{0}\right)\left(1+\frac{p}{\tau}\right), \\
& B_{2}=\left(Q_{2}^{0}\left(P_{1}^{0}+P_{2}^{0}+P_{3}^{0}\right) h-P_{3}^{0}\left(P_{1}^{0}+P_{2}^{0}\right) \frac{h^{3}}{12}\right)\left(1+\frac{p}{\tau}\right), \\
& B_{3}=\left(Q_{1}^{0}\left(P_{1}^{0}+P_{2}^{0}+P_{3}^{0}\right) h+\left(P_{1}^{0}+P_{2}^{0}\right)\left(P_{1}^{0}+P_{2}^{0}+2 P_{3}^{0}\right) \frac{h^{3}}{12}\right)\left(1+\frac{p}{\tau}\right), \\
& B_{4}=Q_{1}^{0}\left(P_{1}^{0}+P_{2}^{0}+P_{3}^{0}\right) h\left(1+\frac{p}{\tau}\right) .
\end{aligned}
$$


As for the Maxwell-type model, we conclude that the two-dimensional constitutive equations of Voigt-type exists as a special case of the three-dimensional viscoelastic behavior, i.e., with the same relaxation parameter $\tau$.

\section{Conclusion}

We have discussed the two-dimensional constitutive equations for resultant force stress and couple stress tensors derived from the constitutive equations of threedimensional viscoelastic Cosserat (micropolar) continuum. The presented results demonstrate how the viscoelastic properties of three-dimensional continuum inherit in the constitutive equations for plates and shells. Within the framework of the linear micropolar viscoelasticity with constitutive equations of differential type we show that 2D relaxation functions of shells have move complicated structures then the relaxation function of the bulk material, in general. In particular, even for homogeneous plates and shells the spectrum of relaxation time do not coincide with the spectrum of the bulk material. For inhomogeneous plates and shells the spectrum may depend also on the structure of the shell in the thickness direction and its curvature in the case of shells.

\section{Appendix: Through-the-thickness integration}

Following [Altenbach and Eremeyev 2009] we present more details on the throughthe-thickness procedure used for the derivation of 2D governing equations of the micropolar theory of plates. Let $V=\left\{(x, y, z) \in \mathbb{R}^{3}:(x, y) \in \mathcal{M} \subset \mathbb{R}^{2}, z \in\right.$ $[-h / 2, h / 2]\}$ be the volume of a plate-like body. We denote the boundary of the plate-like body as $S=S^{\nu} \cup S^{+} \cup S^{-}$, where $S^{ \pm}=\{(x, y, z):(x, y) \in \mathcal{M}, z= \pm h / 2$ are the plate faces and $S^{\nu}=\{(x, y, z):(x, y) \in \mathcal{C} \equiv \partial \mathcal{M}, z \in[-h / 2, h / 2]\}$ is the lateral surface. We consider the following boundary conditions at $S^{ \pm}$

$$
n^{ \pm} \cdot \sigma=t^{ \pm}, \quad n^{ \pm} \cdot \mu=m^{ \pm}
$$

where $\boldsymbol{t}^{ \pm}, \boldsymbol{m}^{ \pm}$are given vector functions, $n^{ \pm}= \pm \boldsymbol{i}_{3}$, and $\boldsymbol{i}_{3}=\boldsymbol{i}_{1} \times \boldsymbol{i}_{2}$.

Integrating $(1)_{1}$ over $[-h / 2, h / 2]$ and introducing the notations

$$
q=\langle\rho f\rangle+t^{+}+t^{-}
$$

we result in (12) 1 with $\mathbf{T}=\langle\mathbf{A} \cdot \boldsymbol{\sigma}\rangle$.

For the derivation of $(12)_{2}$ we cross-multiply $(1)_{2}$ by $z \boldsymbol{i}_{3}$ from the left and again integrate the result through the thickness. Finally, we obtain $(12)_{2}$ with

$$
\boldsymbol{c}=\langle\rho \boldsymbol{l}\rangle+\boldsymbol{m}^{+}+\boldsymbol{m}^{-}+\boldsymbol{i}_{3} \times\langle\rho z \boldsymbol{f}\rangle+\frac{h}{2} \boldsymbol{i}_{3} \times\left(\boldsymbol{t}^{+}-\boldsymbol{t}^{-}\right)
$$

and $\mathbf{M}=\langle\mathbf{A} \cdot \boldsymbol{\mu}\rangle-\left\langle\mathbf{A} \cdot z \boldsymbol{\sigma} \times \boldsymbol{i}_{3}\right\rangle$. 
In the case of linear theory the through-the-thickness integration can be applied to the $3 \mathrm{D}$ constitutive relations with the linear approximation of displacements and rotations

$$
\boldsymbol{u}(x, y, z)=\boldsymbol{v}(x, y)-z \boldsymbol{\phi}(x, y), \quad \boldsymbol{\vartheta}=\boldsymbol{\phi}(x, y) \times \boldsymbol{i}_{3}+\vartheta_{3}(x, y) \boldsymbol{i}_{3},
$$

where $\boldsymbol{\phi} \cdot \boldsymbol{i}_{3}=0$. The approximation (32) is consistent with $2 \mathrm{D}$ equilibrium equations as well as with averaging through the thickness. In particular, 2D fields of translations and rotations can be interpreted as

$$
\boldsymbol{v}=\frac{1}{h}\langle\boldsymbol{u}\rangle, \quad \boldsymbol{\theta}=\boldsymbol{\vartheta} .
$$

Other possible variants of $3 \mathrm{D}$ to $2 \mathrm{D}$ reduction within micropolar elasticity are discussed in [Altenbach et al. 2009; Altenbach and Eremeyev 2009; 2010; Chróścielewski et al. 2011; Sargsyan 2011; Sargsyan and Sargsyan 2014; Steinberg and Kvasov 2013; 2015; Zubov 2009].

\section{Acknowledgements}

Eremeyev was supported by the Russian Foundation of Basic Research with the grant No. 15-01-01492.

\section{References}

[Altenbach and Eremeyev 2009] H. Altenbach and V. A. Eremeyev, "On the linear theory of micropolar plates", ZAMM Z. Angew. Math. Mech. 89:4 (2009), 242-256.

[Altenbach and Eremeyev 2010] H. Altenbach and V. A. Eremeyev, "On the theories of plates based on the Cosserat approach", pp. 27-35 in Mechanics of generalized continua: one hundred years after the Cosserats, edited by G. A. Maugin and A. V. Metrikine, Adv. Mech. Math. 21, Springer, New York, 2010.

[Altenbach et al. 2009] J. Altenbach, H. Altenbach, and V. A. Eremeyev, "On generalized Cosserattype theories of plates and shells: a short review and bibliography”, Arch. Appl. Mech. 80:1 (2009), 73-92.

[Bîrsan and Neff 2013] M. Bîrsan and P. Neff, "Existence theorems in the geometrically non-linear 6-parameter theory of elastic plates", J. Elasticity 112:2 (2013), 185-198.

[Bîrsan and Neff 2014] M. Bîrsan and P. Neff, "Existence of minimizers in the geometrically nonlinear 6-parameter resultant shell theory with drilling rotations", Math. Mech. Solids 19:4 (2014), 376-397.

[Christensen 1971] R. M. Christensen, Theory of viscoelasticity: an introduction, Academic Press, New York, 1971.

[Chróścielewski et al. 2004] J. Chróścielewski, J. Makowski, and W. Pietraszkiewicz, Statyka i dynamika powłok wielopłatowych: nieliniowa teoria i metoda elementów skończonych, Wydawnictwo IPPT PAN, Warsaw, 2004.

[Chróścielewski et al. 2010] J. Chróścielewski, W. Pietraszkiewicz, and W. Witkowski, "On shear correction factors in the non-linear theory of elastic shells", Int. J. Solids Struct. 47 (2010), 35373545 . 
[Chróścielewski et al. 2011] J. Chróścielewski, I. Kreja, A. Sabik, and W. Witkowski, "Modeling of composite shells in 6-parameter nonlinear theory with drilling degree of freedom", Mech. Adv. Mater. Struct. 18 (2011), 403-419.

[Diebels and Steeb 2003] S. Diebels and H. Steeb, "Stress and couple stress in foams", Comput. Mater. Sci. 28 (2003), 714-722.

[Eremeyev and Lebedev 2011] V. A. Eremeyev and L. P. Lebedev, "Existence theorems in the linear theory of micropolar shells", Z. Angew. Math. Mech. 91:6 (2011), 468-476.

[Eremeyev and Zubov 2008] V. A. Eremeyev and L. M. Zubov, Mekhanika uprugikh obolochek, Nauka, Moscow, 2008.

[Eremeyev et al. 2013] V. A. Eremeyev, L. P. Lebedev, and H. Altenbach, Foundations of micropolar mechanics, Springer, Heidelberg, 2013.

[Ericksen and Truesdell 1958] J. L. Ericksen and C. Truesdell, "Exact theory of stress and strain in rods and shells", Arch. Ration. Mech. An. 1 (1958), 295-323.

[Eringen 1999] A. C. Eringen, Microcontinuum field theories, I: Foundations and solids, Springer, New York, 1999.

[Lakes 1986] R. S. Lakes, "Experimental microelasticity of two porous solids", Int. J. Solids Struct. 22 (1986), 55-63.

[Lebedev et al. 2010] L. P. Lebedev, M. J. Cloud, and V. A. Eremeyev, Tensor analysis with applications in mechanics, World Scientific, Hackensack, NJ, 2010.

[Libai and Simmonds 1998] A. Libai and J. G. Simmonds, The nonlinear theory of elastic shells, 2nd ed., Cambridge University Press, 1998.

[Reissner 1977] E. Reissner, "A note on generating generalized two-dimensional plate and shell theories”, Z. Angew. Math. Phys. 28 (1977), 633-642.

[Rubin 2000] M. B. Rubin, Cosserat theories: shells, rods and points, Kluwer, Dordrecht, 2000.

[Sargsyan 2011] S. H. Sargsyan, "The general dynamic theory of micropolar elastic thin shells", Dokl. Phys. 56:1 (2011), 39-42.

[Sargsyan and Sargsyan 2014] A. H. Sargsyan and S. H. Sargsyan, "Dynamic model of micropolar elastic thin plates with independent fields of displacements and rotations", J. Sound Vib. 333:18 (2014), 4354-4375.

[Steinberg and Kvasov 2013] L. Steinberg and R. Kvasov, "Enhanced mathematical model for Cosserat plate bending", Thin-Walled Struct. 63 (2013), 51-62.

[Steinberg and Kvasov 2015] L. Steinberg and R. Kvasov, "Analytical modeling of vibration of micropolar plates", Appl. Math. (SCIRP) 6:5 (2015), 817-836.

[Wilson 1901] E. B. Wilson, Vector analysis: a textbook for the use of students of mathematics and physics, founded upon the lectures of J. Willard Gibbs, Yale University Press, New Haven, 1901. Reprinted by Dover, New York, 1960.

[Zubov 2009] L. M. Zubov, "Micropolar-shell equilibrium equations", Dokl. Phys. 54:6 (2009), 290293.

Received 14 Apr 2015. Revised 12 Aug 2015. Accepted 8 Oct 2015.

Holm Altenbach: holm.altenbach@ovgu.de

Chair of Engineering Mechanics, Institute of Mechanics, Faculty of Mechanical Engineering,

Otto-von-Guericke-Universität, Universitätsplatz 1, 39106 Magdeburg, Germany

VICTOR A. EREMEYEV: eremeyev.victor@gmail.com

Department of Applied Mechanics and Robotics, Rzeszow University of Technology,

al. Powstańców Warszawy, 8, 35-959, Rzeszów, Poland

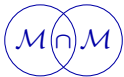


EDITORIAL BOARD

ANTONIO CARCATERRA

ERIC A. CARLEN

FRANCESCO DELL'ISOLA

RAFFAELE ESPOSITO

ALBERT FANNJIANG

Gilles A. FranCFORT

Pierangelo MARCATI

JEAN-JACQUES MARIGO

PETER A. MARKOWICH

MARTIN OSTOJA-STARZEWSKI

PIERRE SEPPECHER

DAVID J. STEIGMANN

PAUl STEINMANN

PierRe M. SuQueT

MANAGING EDITORS

MICOL AMAR

CORRADO LATTANZIO

ANGELA MADEO

MARTIN OSTOJA-STARZEWSKI

ADVISORY BOARD

ADNAN AKAY

Holm AltenBaCH

MICOL AMAR

HARM ASKES

TEODOR ATANACKOVIĆ

VICTOR BERDICHEVSKY

GUY BOUCHITTÉ

ANDREA BRAIDES

ROBERTO CAMASSA

MAURO CARFORE

ERIC DARVE

FELIX DARVE

ANNA DE MASI

GianPiEtro DEL Piero

EMMANUELE Di BENEDETTO

BERNOLD FIEDLER

IRENE M. GAMBA

DAVID Y. GAO

SERGEY GAVRILYUK

TIMOTHY J. HEALEY

DOMINIQUE JEULIN

ROGER E. KHAYAT

CORRADO LATTANZIO

ROBERT P. LIPTON

ANGELO LUONGO

ANGELA MADEO

JUAN J. MANFREDI

CARLO MARCHIORO

GÉRARD A. MAUGIN

ROBERTO NATALINI PATRIZIO NEFF

ANDREY PIATNITSKI

ERRICO PRESUTTI

MARIO PULVIRENTI

LUCIO RUSSO

Miguel A. F. SANJUAN

PATRICK SElVADURAI

ALEXANDER P. SEYRANIAN

MIROSLAV ŠILHAVÝ

GUIDO SWEERS

ANTOINETTE TORDESILLAS

LEV TRUSKINOVSKY

JUAN J. L. VELÁZQUEZ VINCENZO VESPRI ANGELO VULPIANI msp.org/memocs

Università di Roma "La Sapienza", Italia

Rutgers University, USA

(CO-CHAIR) Università di Roma "La Sapienza", Italia

(TREASURER) Università dell'Aquila, Italia

University of California at Davis, USA

(CO-CHAIR) Université Paris-Nord, France

Università dell'Aquila, Italy

École Polytechnique, France

DAMTP Cambridge, UK, and University of Vienna, Austria

(CHAIR MANAGING EDITOR) Univ. of Illinois at Urbana-Champaign, USA

Université du Sud Toulon-Var, France

University of California at Berkeley, USA

Universität Erlangen-Nürnberg, Germany

LMA CNRS Marseille, France

Università di Roma "La Sapienza", Italia

Università dell'Aquila, Italy

Université de Lyon-INSA (Institut National des Sciences Appliquées), France

(CHAIR MANAGING EDITOR) Univ. of Illinois at Urbana-Champaign, USA

Carnegie Mellon University, USA, and Bilkent University, Turkey

Otto-von-Guericke-Universität Magdeburg, Germany

Università di Roma "La Sapienza", Italia

University of Sheffield, UK

University of Novi Sad, Serbia

Wayne State University, USA

Université du Sud Toulon-Var, France

Università di Roma Tor Vergata, Italia

University of North Carolina at Chapel Hill, USA

Università di Pavia, Italia

Stanford University, USA

Institut Polytechnique de Grenoble, France

Università dell'Aquila, Italia

Università di Ferrara and International Research Center MEMOCS, Italia

Vanderbilt University, USA

Freie Universität Berlin, Germany

University of Texas at Austin, USA

Federation University and Australian National University, Australia

Université Aix-Marseille, France

Cornell University, USA

École des Mines, France

University of Western Ontario, Canada

Università dell' Aquila, Italy

Louisiana State University, USA

Università dell'Aquila, Italia

Université de Lyon-INSA (Institut National des Sciences Appliquées), France University of Pittsburgh, USA

Università di Roma "La Sapienza”, Italia

Université Paris VI, France

Istituto per le Applicazioni del Calcolo "M. Picone", Italy

Universität Duisburg-Essen, Germany

Narvik University College, Norway, Russia

Università di Roma Tor Vergata, Italy

Università di Roma "La Sapienza”, Italia

Università di Roma “Tor Vergata”, Italia

Universidad Rey Juan Carlos, Madrid, Spain

McGill University, Canada

Moscow State Lomonosov University, Russia

Academy of Sciences of the Czech Republic

Universität zu Köln, Germany

University of Melbourne, Australia

École Polytechnique, France

Bonn University, Germany

Università di Firenze, Italia

Università di Roma La Sapienza, Italia

MEMOCS (ISSN 2325-3444 electronic, 2326-7186 printed) is a journal of the International Research Center for the Mathematics and Mechanics of Complex Systems at the Università dell'Aquila, Italy.

Cover image: "Tangle” by $\odot$ John Horigan; produced using the Context Free program (contextfreeart.org).

PUBLISHED BY

7 mathematical sciences publishers

nonprofit scientific publishing

http://msp.org/

(C) 2015 Mathematical Sciences Publishers 
Mathematics and Mechanics of Complex Systems vol. 3 no. 3

Stationary solutions of Keller-Segel-type crowd motion and 211 herding models: Multiplicity and dynamical stability

Jean Dolbeault, Gaspard Jankowiak and Peter

Markowich

Comprehensive description of deformation of solids as 243 wave dynamics

\section{Sanichiro Yoshida}

On the constitutive equations of viscoelastic micropolar 273 plates and shells of differential type

Holm Altenbach and Victor A. Eremeyev

Identification of higher-order elastic constants for grain 285 assemblies based upon granular micromechanics

Anil Misra and Payam Poorsolhjouy

MEMOCS is a journal of the International Research Center for the Mathematics and Mechanics of Complex Systems at the Università dell' Aquila, Italy.

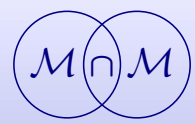

